\title{
Autonomous Systems and Artificial Intelligence - Hype or Prerequisite for P5 Medicine?
}

\author{
Bernd BLOBEL $^{\mathrm{a}, \mathrm{b}, \mathrm{c}, 1}$, Pekka RUOTSALAINEN ${ }^{\mathrm{d}}$, Mathias BROCHHAUSEN ${ }^{\mathrm{e}}$ \\ ${ }^{a}$ Medical Faculty, University of Regensburg, Germany \\ ${ }^{\mathrm{b}}$ eHealth Competence Center Bavaria, Deggendorf Institute of Technology, Germany \\ ${ }^{\mathrm{c}}$ First Medical Faculty, Charles University Prague, Czech Republic \\ ${ }^{\mathrm{d}}$ Faculty of Information Technology and Communication Sciences (ITC), Tampere \\ University, Finland

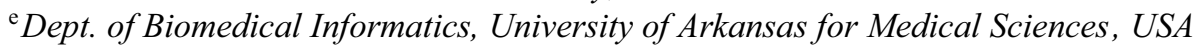

\begin{abstract}
For meeting the challenge of aging, multi-diseased societies, cost containment, workforce development and consumerism by improved care quality and patient safety as well as more effective and efficient care processes, health and social care systems around the globe undergo an organizational, methodological and technological transformation towards personalized, preventive, predictive, participative precision medicine (P5 medicine). This paper addresses chances, challenges and risks of specific disruptive methodologies and technologies for the transformation of health and social care systems, especially focusing on the deployment of intelligent and autonomous systems.
\end{abstract}

Keywords. Healthcare transformation, pHealth, Artificial intelligence, Autonomous systems, Learning, Knowledge representation, Knowledge management, Ethics

\section{Introduction}

Personalized medicine individualizes diagnoses and treatments according to the personal health status, genetic, environmental, occupational, and social conditions and context by understanding the pathology of diseases including the individual predisposition to diseases and responsiveness to treatment. For understanding a disease's pathology and undertaking scientifically sound predictions and preventions, we have to explore the mechanisms and processes from molecule up to society, transferring basic sciences and biomedical research into clinical practice, adding precision medicine to the approach. Thereby, all interacting factors and components impacting individuals' health, such as genomes, epigenomes, proteomes, microbiomes, metabolomes, pharmacomes, transcriptomes, cognitive-affective behavioromes, etc., summarized as interactomes, must be considered. The entire approach requires a massive involvement of the subject of care and/or his/her social environment, extending the approach to participative health. The resulting personalized, preventive, predictive, participative precision medicine (P5

${ }^{1}$ Corresponding Author: Bernd Blobel, PhD, FACMI, FACHI, FHL7, FEFMI, FIAHSI, Professor; Medical Faculty, University of Regensburg, Germany; Email: bernd.blobel@klinik.uni-regensburg.de 
medicine) allows containing costs despite aging and multi-diseased societies, improving care quality and safety, and managing health and social services consumerism. P5 medicine requires the involvement of multiple domains and disciplines with their own methodologies, languages, terminologies and ontologies, such as systems medicine, biology, physics up to the quantum level, chemistry, bioinformatics, genomics, but also social sciences, public health, etc., resulting in an incredible and continuously growing amount of data and information. Table 1 summarizes objectives, characteristics and methodologies as inevitable prerequisites for transforming health and social care systems [1].

Table 1. Transformed health ecosystems' objectives and characteristics as well as methodologies for meeting them, after [1]

\begin{tabular}{|c|c|c|}
\hline Objective & Characteristics & Methodologies \\
\hline $\begin{array}{l}\text { Provision of health services } \\
\text { everywhere anytime }\end{array}$ & $\begin{array}{ll}- & \text { Openness } \\
\text { - } & \text { Distribution } \\
\text { - } & \text { Mobility } \\
\text { - } & \text { Pervasiveness } \\
\text { - } & \text { Ubiquity }\end{array}$ & $\begin{array}{ll}\text { - } & \text { Wearable and implantable } \\
\text { - } & \text { Pensors and actuators } \\
\text { - } & \text { and network connectivity } \\
\text { - } & \text { Embedded intelligence } \\
\text { - } & \text { Context awareness }\end{array}$ \\
\hline $\begin{array}{l}\text { Individualization of the system } \\
\text { according to status, context, } \\
\text { needs, expectations, wishes, } \\
\text { environments, etc., of the subject } \\
\text { of care }\end{array}$ & $\begin{array}{ll}\text { - } & \text { Flexibility } \\
\text { - } & \text { Scalability } \\
\text { - } & \text { Cognition } \\
\text { - } & \text { Affect and Behavior } \\
\text { - } & \text { Autonomy } \\
\text { - } & \text { Adaptability } \\
\text { - } & \text { Self-organization } \\
\text { - } & \text { Subject of care } \\
\text { - } & \text { involvement } \\
\end{array}$ & $\begin{array}{ll}\text { - } & \text { Personal and environmental } \\
\text { - } & \text { data integration and analytics } \\
\text { - } & \text { Corvice integration } \\
\text { - } & \text { Knowledge integration } \\
\text { - } & \text { Process and decision } \\
\text { - } & \text { intelligence } \\
\text { Presentation layer for all } \\
\text { actors }\end{array}$ \\
\hline $\begin{array}{l}\text { Integration of different actors } \\
\text { from different disciplines/do- } \\
\text { mains (incl. the participation/ } \\
\text { empowerment of the subject of } \\
\text { care), using their own languages, } \\
\text { methodologies, terminologies, } \\
\text { ontologies, thereby meeting any } \\
\text { behavioral aspects, rules and } \\
\text { regulations }\end{array}$ & $\begin{array}{ll}- & \text { Architectural framework } \\
- & \text { End-user interoperability } \\
- & \text { Management and } \\
\text { harmonization of multiple } \\
\text { domains including policy } \\
\text { domains }\end{array}$ & $\begin{array}{l}\text { Terminology and ontology } \\
\text { management and } \\
\text { harmonization } \\
\text { - } \quad \text { Knowledge harmonization } \\
\text { - Language transformation/ } \\
\text { translation }\end{array}$ \\
\hline $\begin{array}{l}\text { Usability and acceptability of } \\
\text { pHealth solutions }\end{array}$ & $\begin{array}{ll}\text { - } & \text { Preparedness of the } \\
\text { individual subject of care } \\
\text { Security, privacy and trust } \\
\text { framework } \\
\text { - } \quad \text { Consumerization } \\
\text { - } \quad \text { Subject of care } \\
\text { empowerment } \\
\text { - } \quad \text { Subject of care as manager } \\
\text { Information based } \\
\text { assessment and selection of } \\
\text { services, service quality } \\
\text { and safety as well as } \\
\text { trustworthiness } \\
\text { Lifestyle improvement and } \\
\text { Ambient Assisted Living } \\
\text { (AAL) services }\end{array}$ & $\begin{array}{ll}\text { - } & \text { Tool-based ontology } \\
\text { management }\end{array}$ \\
\hline
\end{tabular}


For collecting, managing and using those data, new techniques and methods have to be exploited such as mobile, bio-, nano- molecular technologies, big data and analytics, advanced computing, virtual reality, learning algorithms, etc. An overview on technologies and methodologies enabling P5 medicine is presented in Table 2 [2].

Table 2. Technologies and methodologies for transforming health ecosystems [2]

\begin{tabular}{|c|c|}
\hline $\begin{array}{ll}\text { - } & \text { Mobile technologies, biotechnologies, nano- } \\
\text { and molecular technologies } \\
\text { - } \quad \text { Big data and business analytics } \\
\text { - } \quad \text { Antegration of analytics and apps } \\
\text { Assisting technologies } \rightarrow \text { Robotics, } \\
\text { autonomous systems } \\
\text { Natural Language Processing } \rightarrow \text { Text } \\
\text { analytics } \rightarrow \text { Intelligent media analytics } \\
\text { Conceptualization } \rightarrow \text { Knowledge } \\
\text { management (KM) and knowledge } \\
\text { representation (KR) } \rightarrow \text { Artificial intelligence } \\
\text { (AI) } \rightarrow \text { Artificial common (general) } \\
\text { intelligence } \rightarrow \text { Intelligent autonomous } \\
\text { systems } \\
\text { Security and privacy, governance, ethical } \\
\text { challenges, Education } \rightarrow \text { Ethical AI } \\
\text { Principles } \\
\text { Cloud computing, cognitive computing, social } \\
\text { business }\end{array}$ & $\begin{array}{l}\text { - Edge computing as a "family of technologies } \\
\text { that distributes data and services where they } \\
\text { best optimize outcomes in a growing set of } \\
\text { connected assets“ (Forrester Research) } \\
\text { - Virtual reality and augmented reality, thereby } \\
\text { blurring "the boundaries between the physical } \\
\text { and digital worlds" (Gartner) } \\
\text { - Creation of IoT-Platforms and app-ecosystems } \\
\text { - Patient-generated health data ecosystem } \rightarrow \\
\text { multiple, dynamic policies } \\
\text { - Web content management } \rightarrow \text { Digital experience } \\
\text { management } \\
\text { Data bases } \rightarrow \text { NoSQL technologies } \rightarrow \text { Data } \\
\text { warehouses } \rightarrow \text { Graph DBs } \rightarrow \text { Data lakes } \\
\text { - EHR extension with genomic data } \\
\rightarrow \text { Specifications } \rightarrow \text { Implementation } \rightarrow \text { Tooling } \\
\rightarrow \text { Testing } \rightarrow \text { Certification }\end{array}$ \\
\hline
\end{tabular}

Use of artificial intelligence (AI) technologies for health holds great promise and has already contributed to important advances in fields such as drug discovery, genomics, radiology, pathology and prevention. AI could assist health-care providers in avoiding errors and allow clinicians to focus on providing care and solving complex cases. Further details on health transformation can be found in [3].

\section{Methods}

Transformation of health and social services according to the P5 medicine paradigm results in highly complex and highly dynamic multi-disciplinary systems, which have to be context-sensitive and cognitive to represent the intended settings in structure and function correctly and consistently. We do not have workforce, skills and power to manage such systems manually anymore. This holds for data search, collection, interrelation, interpretation and processing, but also for designing and managing the underlying complex and domain-crossing processes, not talking about the knowledge representation and management challenges discussed before and in [4]. Furthermore, we cannot place specialists next to every person to be ubiquitously cared for. Therefore, the deployment of robotics and artificial intelligence, or more generally autonomous and intelligent systems (AIS), using machine learning, big data and analytics at different levels is inevitable. Focusing on autonomous and intelligent systems in general regarding their cognitive functions, in this paper we will not address the specific properties of robots physically interacting with its environment.

Intelligence is a concept in cognition theory with four foundational principles: data, information, knowledge, and wisdom [5]. During investigations and observations, organs or sensors collect data as measures or symbols describing the world, that way forming 
the structural level of intelligence. By attaching meaning to data, they are then transformed into information for taking decisions, establishing the semantic level of intelligence. Knowledge enables proper actions on the represented system, supervised and evaluated by wisdom. More background information on knowledge representation and intelligence can be found in [2].

The approach to artificial intelligence (AI) as used today was originated in 1950 by Alan Turing [6]. Considering AI, many definitions exist. McKinsey defines AI as "the ability of a machine to perform cognitive functions we associate with human minds, such as perceiving, reasoning, learning, and problem solving" [7]. It represents an interdisciplinary approach including mathematics, logics, cognitive sciences, life sciences, but also computation and engineering to manage processes such as modeling, simulating, understanding, etc. [8]. A simpler definition characterizes AI as ability of machines to simulate human intelligence [9]. The OECD provides in its Recommendation of the Council on Artificial Intelligence a specific AI definition addressing main aspects of this paper as follows. "An AI system is a machine-based system that can, for a given set of human-defined objectives, make predictions, recommendations, or decisions influencing real or virtual environments. AI systems are designed to operate with varying levels of autonomy." [10]. Autonomy and automation levels are non-autonomous (assistive) or full autonomous refined by multiple risks levels, and conditional automation, high automation, and full automation, respectively [11].

AI and automation in healthcare aims at augmentation of capabilities and engagement for care provider and subject of care including education, access to information and services, etc. It enables cooperation, improves staff and patient experiences, but also processes including clinical workflow and scheduling, business efficiency, productivity and cost containment as well as risk analysis. Furthermore, it facilitates faster and more precise decisions of direct and indirect caregivers, administrators and patients including prognosis. Finally, it enables collaborative business intelligence as self-service. Therefore, we can distinguish different levels of intelligence in AI such as assisted intelligence automating tasks like pattern recognition with human input and intervention, augmented intelligence combining existing information with predetermined solutions based on different levels of machine learning, and autonomous intelligence deploying genetic algorithms and evolutionary strategies to act independently from humans [12].

Application domains of AI in healthcare are for example AI in medical imaging, AI in digital pathology, $\mathrm{AI}$ in genomics, AI for understanding and predicting the course of a disease, etc. The aforementioned application domains require different levels of AI from machine learning through deep learning up to swarm learning and are closely associated with big data, so representing the different levels of intelligence introduced before [13]. Most of current AI applications follow the weak AI (narrow AI) approach, usually just replicating human intelligence in the specific context such as simple classification, pattern recognition and assistive systems. Here, facial recognition, conversational assistants and chatbots, but also recommendation engines are well-known applications. The concept of artificial general intelligence (AGI) (sometimes also called strong intelligence) aims at mimicking the full range of human cognitive and intellectual capabilities, resulting in autonomous systems [14]. Artificial superintelligence (ASI) goes even beyond this approach by exceeding human intellectual power, almost comprehensively covering all categories and fields of endeavor [15]. Another way to classify AI refers to its implementation (in bracket equalizing the levels with the classification provided before). At lowest level we find reactive AI systems, followed by 
limited memory machines (narrow intelligence) using historical experiences to inform future decisions, theory of mind AI systems (AGI) to infer intentions and predict behavior, and finally self-aware AI systems (ASI) [16]. Technologies for enabling AI, summarized in Table 2, are for example robots, virtual agents, computer vision and virtual reality, analytics, machine learning and natural language processing, understanding and generation.

Data science incorporates various disciplines -- for example, data engineering, data preparation, data exploration, data mining, predictive analytics, machine learning and data visualization, as well as mathematics, statistics and software programming. A predominant challenge data science addresses is the elimination of bias in data sets and analytics applications [17].

Data analytics can be provided at different levels. The first level is descriptive analytics, presenting what has happened. The next level is predictive analytics, providing insights regarding what will happen. The highest level is prescriptive analytics, providing foresights by defining how to perform to make things/processes happen. More details on analytics, its adoption model and related issues can be found in [4].

Machine learning as subset of artificial intelligence is at its most basic the practice of using algorithms to parse data, learn from it, and then make a determination or prediction about something in the world. Here we distinguish between supervised learning training algorithms with human-labeled data, unsupervised learning inferring some structure from unlabeled data, and reinforcement learning deploying algorithms rewarding overcoming mistakes. In the latter case, Markov decision processes are typically used. Deep learning is a subset of machine learning that processes data using multi-layer neural networks, leveraging learning algorithms that mimic the function of the human brain. Specializations of neural networks are recurrent neural networks (RNNs) used in speech recognition and natural language processing by pattern analysis to predict the next likely object or scenario. Convolutional neural networks (CNNs) deployed in computer vision belong to the same neural network class [18]. Swarm learning is the newest approach using AI at the edge by decentralizing the analysis of data from multiple locations and sharing insights while protecting data sovereignty [19, 20]. When creating new knowledge by properly modeling a system in question, we have to validate the outcome on the real world system and thereafter adopted the knowledge representation if needed [21]. This holds for human-made and AI mediated knowledge representation development processes.

Modern AI applications rely more on learning from data to discover possibly new knowledge that needs verification than just codifying existing knowledge to automate problem solving. Thereby, they are facing two challenges. First, the solution might suffer from the lack of sound explainability compared with an established knowledge framework. Second, it might incorporate bias or errors due to biased or poor data the application has learned from [22]. Possible sources of bias are, e.g., insufficient data, skewed data, limited features, historical bias or unreliable labels, proxies [23].

\section{Ethical Challenges of Artificial Intelligence and Autonomous Systems}

Any action and relationship in enlightened democratic societies, and especially in health and social care ecosystems have to accommodate legal, moral and ethical principles. Contracts and law define and enforce behavior for maintaining relations, peace, and justice in a society. Ethics provides code and conduct guiding to decide what is good or 
bad, and how to act and behave properly. It establishes and defends rules of morality, which frequently go beyond or contradicts the law [4, 24]. Ethical values are strongly impacted by culture, social norms and geographic locations. With the evolution of societies including sciences and technologies, approaches to ethics and its underlying theories in the framework of meta-ethics, normative ethics and applied ethics show evolutionary characteristics as well. Examples of this evolution are Aristotle's and Plato's virtue ethics, Kant's deontological ethics, Mill's utilitarian ethics, and Rawls' justice as fairness ethics [24]. In the special context of autonomous and intelligent systems for health and social care, we have also to mention consequentialism ethics, raising questions about acceptable consequences to the individual, or how to balance personal and benefits. Therefore, it is impossible to implement one global comprehensive standard of ethics. Instead, basic social and ethical principles such as dignity, freedom, autonomy, privacy, equality and solidarity, or the more technological categories like fairness, robustness, explainability and lineage have been established by different organization for different domains. In the context of AIS, such classification is more relevant than a philosophical one. The different sets of principles provided by governmental and non-governmental organizations as well as by vendors will be briefly discussed in the following chapter.

\section{Ethical Frameworks}

The four traditional bioethical principles are autonomy, beneficence, non-maleficence, and justice. For building public confidence in disruptive technologies, promoting safer practices and to facilitating broader societal adoption, explicability should be added. [25]. For designing and managing AIS solutions, following principles must be considered: Fair Information Principles [26]; Fair Information Practice Principles [27]; Ethical Principles; Big Data Best Privacy Practices according to the aforementioned Federal Trade Commission (FTC) Guidelines [27]. Digital change requires zero trust and a changed role of Chief Information Security Officers (CISOs) / Chief Privacy Officers (CPOs) - from security management to risk management, but also the inclusion of newer ethical initiatives. AI ethics according to IBM's definition aims at optimizing AI's beneficial impact while reducing risks and adverse outcomes for all stakeholders. It also involves identifying, studying, and proposing technical and nontechnical solutions for ethics issues arising from the pervasive use of AI in life and society. Examples of such issues are data responsibility and privacy, fairness, inclusion, moral agency, value alignment, accountability, transparency, explainability, trust, robustness and, awareness of technology misuse. [28, 29].

The WHO normative guidance "Ethics and governance of artificial intelligence for health" requests putting ethical considerations and human rights at the center of the design, development, and deployment of AI technologies for health, that way also fighting digital divide locally (exclusion of populations) and globally (low- and middleincome countries). Challenges to be met are the establishment of key ethical principles for the use of AI for health; the protection of human autonomy; the promotion of human well-being and safety and the public interest; ensuring transparency, explainability and intelligibility; fostering responsibility and accountability; ensuring inclusiveness and equity; and the promotion of AI that is responsive and sustainable [30]. That way, WHO responds on pioneering developments in fields such as genomics, epigenetics, gene 
editing, artificial intelligence, and big data, all of which pose transformational opportunities but also risks to global health.

In its Global Initiative on Ethics of Autonomous and Intelligent Systems, IEEE defines the following success factors: Participatory design, consensus, multiple discipline focus, recognition of the socio-technical, and focus on design. [31, 32]. AI principles supporting IEEE Ethically Aligned Design include: Sustainable development, Well-being; Human-centered values; Fairness; Transparency and explainability; Robustness, security and safety; Accountability. The first author is actively involved in several projects of the IEEE 7000 Series "Ethics in Action in Autonomous and Intelligent Systems" [33].

The OECD proposed in the Conference Toward AI Network Society, April 2015, in Japan some years ago already Principles for AI Research and Development such as: Transparency; User Assistance; Control-ability; Security; Safety; Privacy; Ethics; and Accountability. The principles resulted in a related guideline prepared by the conference host Japan [34].

The World Economic Forum published the following Top Ethical Issues in Artificial Intelligence [35] to be addressed: Unemployment; Inequality; Humanity; Artificial stupidity; Racist robots; Security; Evil genies; Singularity; Robot rights.

The European Commission recently released a proposal for a regulation of the European Parliament and the Council laying down harmonized rules on artificial intelligence (Artificial Intelligence Act) and amending certain EU legislative acts [36]. The project aims at guaranteeing that AI systems placed on the Union market and used are safe and respect existing law on fundamental rights and Union values such as data protection, consumer protection, non-discrimination and gender equality.

Google established the following 6 objectives for AI applications: be socially beneficial, avoid creating or reinforcing unfair bias, be built and tested for safety, be accountable to people, incorporate privacy design principles, uphold high standards of scientific excellence [37].

Further ethical frameworks are The Asimolar AI Principles of the Future of Life Institute [38]; the Congress Resolution Supporting the Development of Guidelines for the Ethical Development of Artificial Intelligence [39]; and the BS 8611:2016 Robots and Robotic Devices. Guide to the Ethical Design and Application of Robots and Robotic Systems [40]. More information can be found in [4, 41].

Table 3 summarizes the essence of the different ethical frameworks.

Table 3. Common A/IS principles proposed by different organizations

\begin{tabular}{|c|c|c|c|c|c|c|c|c|c|}
\hline 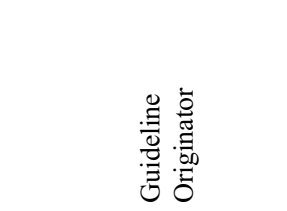 & 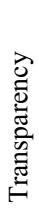 & 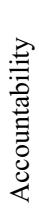 & 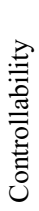 & 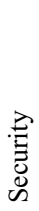 & 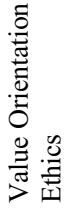 & 总 & 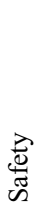 & $\frac{y}{\tilde{a}}$ & 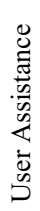 \\
\hline OECD & $\mathrm{x}$ & $\mathrm{x}$ & $\mathrm{x}$ & $\mathrm{x}$ & $\mathrm{x}$ & $\mathrm{x}$ & $\mathrm{x}$ & & $\mathrm{x}$ \\
\hline IEEE & $\mathrm{x}$ & $\mathrm{x}$ & $\mathrm{x}$ & & $\mathrm{X}$ & $\mathrm{x}$ & & $\mathrm{x}$ & $\mathrm{x}$ \\
\hline Asilomar & $\mathrm{x}$ & $\mathrm{x}$ & $\mathrm{x}$ & $\mathrm{x}$ & $\mathrm{x}$ & $\mathrm{x}$ & $\mathrm{x}$ & $\mathrm{x}$ & \\
\hline US Congress & $\mathrm{x}$ & $\mathrm{x}$ & $\mathrm{x}$ & $\mathrm{x}$ & & $\mathrm{x}$ & $\mathrm{x}$ & & $\mathrm{x}$ \\
\hline World Economic Forum & & & & $\mathrm{x}$ & $\mathrm{x}$ & & & $\mathrm{x}$ & \\
\hline
\end{tabular}




\section{Discussion}

The different application domains, managing the different objectives of transformed pHealth ecosystems with different methodologies (Table 1), require different technologies (Table 2) including computing technologies and computing power.

At simplest level, we program a computer to perform a specific task without learning needed at computer side. This approach was followed by the deployment of specific or generalized models of statistics including probabilistic reasoning for automated medical diagnosis. An example is monitoring (recording and assessing) vital signs in the context of home care or ambient assisted living (AAL) supported by any type of portable devices such as smart watches, realized by nowadays microprocessors.

Machine learning focuses on prediction of known properties learned from training data, e.g. codifying and mapping input and output data patterns (existing knowledge) as pattern recognition by supervised learning, while data mining focuses on discovery of unknown properties, e.g. discovering hidden data patterns (new knowledge discovery) by unsupervised learning. Machine learning as optimization reduces the loss in training data sets by comparing predictions with current instances. Machine learning as generalization towards deep learning minimizes unknown properties, moving from data model to algorithmic model.

For assessing impacts of the aforementioned individual interactomes on the predisposition to a disease and responsiveness to treatment, a multidisciplinary approach to a complex, dynamic system is inevitable. As the specific structure and behavior of the considered system and its relations is unknown, a deep learning approach with advanced neural networks is necessary, requiring strong computing power.

When considering complex genotype-phenotype interactions for understanding the detailed pathology of diseases, analyzing and predicting the protein structure in the context of cell mutation and treatment in cancer or for developing vaccines and predicting their pharmacology, the deployment of evolutionary (genetic) algorithms on super computers or even quantum computers is necessary. The same holds for integrating individual health aspects and public health strategies. When creating new knowledge, its verification requires interactions with reality and therefore cognitive computing. Cognitive computing systems consider their environment and dynamically process huge amount of data from multiple and variant sources at high speed. For that purpose, they have to be adaptive, interactive, contextual, iterative and stateful [42]. A shift to cognitive computing is occurring in omics-driven biotechnology to enable precision medicine [43]. Cognition goes beyond recognition and includes knowledge and understanding.

The growing complexity, flexibility and dynamics of pHealth ecosystems as well as new technologies make it harder to maintain governance, security and privacy. Therefore, AIS must also be developed and implemented to maintain those important principles.

For modeling transformed health ecosystems, we have to represent the multiple domains contributing to the health and social care process, but also the rules to defining the behavior of the systems, summarized as policy domain. This domain can be refined into sub-policy domains such as process policies, legal policies, contextual policy (conditions and preferences influencing, e.g., privacy decisions) or ethical policies. For correctly and consistently integrating and interrelating multiple domains, they have to be modeled using the ISO 23903 Interoperability and Integration Reference Architecture [44]. This standard allows to model different knowledge spaces by architecturally representing the related domains and representing them through the domains ontologies. This requires representing the ethical domain by an ethical domain ontology, which has 
been recently developed by the IEEE 7007 project the first author is member of [45]. In addition, key components of ethical considerations, such a deontic roles, rights and obligations, have been represented in the Document Act Ontology (d-acts) [46, 47]. Figure 1 shows the ISO 23903 base model (a) and its instantiation for the policy domains relevant in the context of autonomous and intelligent systems (b).

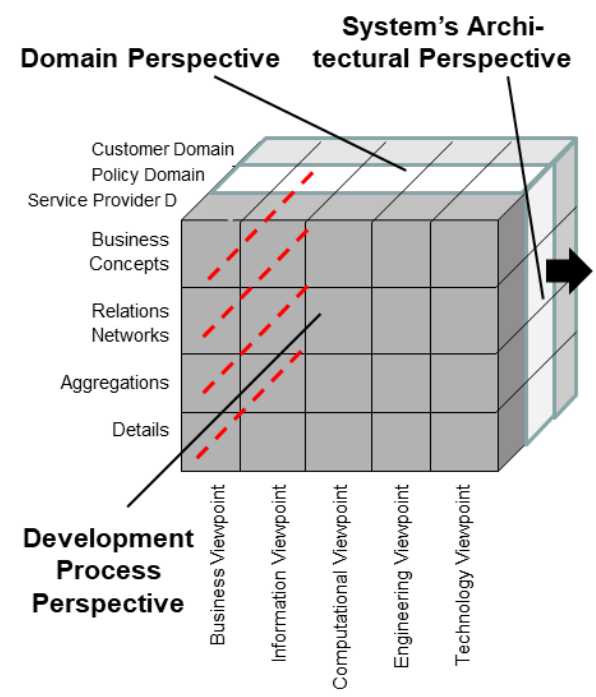

a

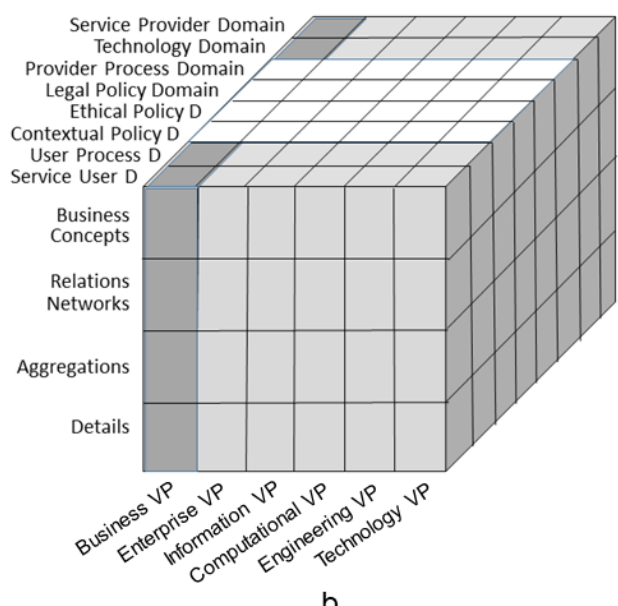

b

Figure 1. AIS representation, design and implementation enabling advanced interoperability and integration acc. to ISO 23903

\section{Conclusion}

Artificial and intelligent systems have a huge potential for strengthening the delivery of health and social services everywhere and anytime, achieving universal health coverage including low- and middle-income countries, but it also poses risks to global health and wellbeing. So it can cause or strengthen digital divide between rich and low- and middleincome countries, but also in developed countries. This could be reasoned by available resources, but also by gender, geography, culture, religion, language, or age. For guaranteeing the promised benefits for public health and medicine when designing, developing and deploying AIS, moral and ethical considerations as well as human rights must play a central role [30].

P5 medicine without artificial intelligence and autonomous systems is not feasible, but we have to advance ourselves to do this right. Hereby, objectives, basic principles, limitations, etc., must be carefully considered and defined in their economic, social, political and environmental context.

Innovations in science and technology are always bound to new social, moral and ethical challenges [48]. With AIS, we can do good things, but also wrong things at all, faster and stricter. Ethical issues could be the misuse of personal information or misinformation and deep fakes, but also lacking oversight and the acceptance of responsibility. Moreover, advanced neurotechnology could change behavior or thought patterns, affecting privacy and dignity. Genetic engineering could overcome damaging 
genetic mutations, but also create new pandemic viruses. While possessing great potential for human health and the recovery from damaging genetic mutations, there are considerable ethical considerations that surround the editing of the human genome. A crucial aspect of new technologies is they weaponization, as discussed in the context of killer robots and combat drones. Ashley Watters raised the question: "At what point do we trust our technology to fight a war for us?" [48].

Our social, moral and ethical decisions are strongly impacted by our underlying value system. Current core aspects are individual freedom with a tendency to ethical egoism, materialism, profit orientation. Contrary to other domains such as manufacturing, trading, consumption, etc., which can be managed with market economy principles such as supply and demand, profit maximization by cost-benefit minimization, etc., realized according to opportunities and choices, the request for health services is usually defined by objective needs. The global health coverage needs contradicting the aforementioned wrong principles. Health services should not be first seen as business opportunities, but as responsibilities, care and duties practiced. The market- and profit-driven global economy puts us in danger regarding security and safety aspects (availability and safety of products), clearly demonstrated during the recent COVID-19 pandemics. Therefore, the European Union decided to re-organize its economy by reducing dependencies from other regions such as India or China. Another example is the state health and social insurance system as fiduciary duty, introduced in the eighties of the $19^{\text {th }}$ century by the German Emperor as implementation of basic ethical concerns raised in the enlightenment period. Meanwhile, this approach has been partially eroded by opening the field to private players for market orientation and competitions. As another of many further examples from all around the world, the recurring discussion on moves such as Obama Care from an ideological instead of moral-ethical perspective demonstrate misconceptions and lack of virtue and acceptance of responsibility.

Considering the financial pressure health systems face to increase income and reduce expenditures, resulting even in inappropriate diagnostic and treatment procedures just because they are more expensive than traditional practices (e.g. CT and MRI instead of auscultation, or surgery instead of physiotherapy). Recently, the American College of Physicians (ACP) criticized in a paper published in the Annals of Internal Medicine the increasing dominance of the profit motive in medicine [49]. It cites Thomas G. Cooney, Chair, Board of Regents, ACP, with "We need to be sure that profits never become more important than patient care in the practice of medicine."

In consequence, we have to ethically revise and redesign our health ecosystem. A good moral, humanistic, social and ethical, but also transparent and quality-controlled approach to autonomous and intelligent systems in health and social care covers the full spectrum from the individual obliged to act in good faith, serves the society, protect the earth and understands the universe, mirroring the continuum addressed with the P5 medicine paradigm. That way, we would reactivate and advance the humanistic, cognitive and logical principles of the Enlightening period with ethical and moral codes of conduct, thereby establishing core values such as virtue, equality, integrity, solidarity, respect, faith and truth. We have to go clearly beyond the fiduciary duties defining most ecosystems' behavior today. In that context, we could also learn from the Committee on Standards in Public Life in Great Britain, which defined 7 ethical principles [50]. Those principles are selflessness, integrity, objectivity, accountability, openness, honesty, and leadership, acknowledging that principles alone cannot guarantee ethical AI [51].

Learning from our practice and reflecting our principles, autonomous and intelligent systems result in faster and stricter innovations and evolutions of transformed health 
ecosystems both in good and in bad faith. It is up to us to reshape our reality to strengthen the benefits and reducing the risks of the new technologies in the ongoing transformation of health and social care systems.

\section{Acknowledgement}

The authors are indebted to thank their colleagues from HL7, ISO TC 215 and CEN TC 251 for their kind and constructive support and cooperation.

\section{References}

[1] Blobel B, Ruotsalainen P, Lopez DM, Oemig F. Requirements and Solutions for Personalized Health Systems. Stud Health Technol Inform. 2017; 237: 3-21.

[2] Blobel B, Ruotsalainen P, Oemig F. Why Interoperability at Data Level Is Not Sufficient for Enabling pHealth? Stud Health Technol Inform. 2020; 273: 3-19.

[3] Blobel B. Challenges and Solutions for Designing and Managing pHealth Ecosystems. Front. Med. 2019; 6: 83. doi: 10.3389/fmed.2019.00083

[4] Blobel B, Ruotsalainen P. Healthcare Transformation Towards Personalized Medicine - Chances and Challenges. Stud Health Technol Inform. 2019; 261: 3-21.

[5] Makhfi P. Introduction to Knowledge Modeling. MAKHFI.com: www.makhfi.com/KCM_intro.htm

[6] Turing A. Computing Machinery and Intelligence, Mind, October 1950; LIX (236): 433-460, doi:10.1093/mind/LIX.236.433, ISSN 0026-4423

[7] McKinsey. An executive's guide to AI. McKinsey Insights 2020. https://www.mckinsey.com/businessfunctions/mckinsey-analytics/our-insights/an-executives-guide-to-ai

[8] Guan J. Artificial Intelligence in Healthcare and Medicine - Promises, Ethical Challenges and Governance. Chin Med Sci J. 2019; 34 (2): 76-83.

[9] General Electric Company. AI in Healthcare: Keys to a Smarter Future. GE Healthcare 2018.

[10] Organisation for Economic Co-operation and Development. Recommendation of the Council on Artificial Intelligence. OECD/LEGAL/0449, 2019. http://legalinstruments.oecd.org.

[11] Bitterman DS, Aerts HJWL, Mak RH. Approaching autonomy in medical artificial intelligence. Lancet Digit Health. September 2020; 2(9): e447-9.

[12] Defining the Roadmap - The Age of Intelligence: Matching Mind and Machine. AI in Healthcare. Pure Storage, Summer 2018.

[13] Ahuja S, McNamara M. AI in Healthcare - Smart Infrastructure Choices Increase Success. NVIDIA, November 2019

[14] Schmelzer R. Data science vs. machine learning vs. AI - How they work together. TechTarget, Business Analytics, 29 August 2021. https://searchbusinessanalytics.techtarget.com/feature/Data-science-vsmachine-learning-vs-AI-How-they-work-together

[15] Wigmore I. artificial superintelligence (ASI). TechTarget, EnterpriseAI, February 2018. https://searchenterpriseai.techtarget.com/definition/artificial-superintelligence-ASI

[16] Tucci L. A guide to artificial intelligence in the enterprise. TechTarget, EnterpriseAI. 9 July 2021. https://searchenterpriseai.techtarget.com/Ultimate-guide-to-artificial-intelligence-in-the-enterprise

[17] Stedman C. What is Data Science? The Ultimate Guide. TechTarget; EnterpriseAI, August 2021. https://searchenterpriseai.techtarget.com/definition/data-science

[18] Laskowski N. recurrent neural networks. TechTarget, EnterpriseAI, July 2021. https://searchenterpriseai.techtarget.com/definition/recurrent-neural-networks

[19] Behr A. Advancing medicine with AI at the edge. Hewlett Packard Enterprise Company, 11 May 2021. https://www.hpe.com/us/en/insights/articles/advancing-medicine-with-ai-at-the-edge-2105.html

[20] Chernicoff D. What's all the buzz about swarm learning. Hewlett Packard Enterprise Company, 11 May 2021. https:/www.hpe.com/us/en/insights/articles/whats-all-the-buzz-about-swarming-learning2108.html

[21] Doerner H. Knowledge Representation. Ideas - Aspects - Formalisms. In: Grabowski J, Jantke KP, Thiele H (Edrs). Foundations of Artificial Intelligence. Berlin: Akademie-Verlag; 1989.

[22] Fridsma DB. Response of the American Medical Informatics Association on FDA-2019-N-1185; "Proposed Regulatory Framework for Modifications to Artificial Intelligence/Machine Learning 
(AI/ML)-Based Software as a Medical Device (SaMD) - Discussion Paper and Request for Feedback". AMIA, 3 June 2019.

[23] DataRobot. Trusted AI 102: A Guide to Building Fair and Unbiased AI Systems. https://www.datarobot.com/resources/trusted-ai-102-fairness-and-bias/

[24] Tzafestas SG. Ethics and Law in the Internet of Things. Smart Cities 2018; 1(1): 98-120.

[25] Ursin F, Timmermann C, Steger F. Explicability of artificial intelligence in radiology - Is a fifth bioethical principle conceptually necessary? Bioethics July 2021; 00: 1-11. doi: 10.1111/bioe.12918. https://onlinelibrary.wiley.com/doi/full/10.1111/bioe.12918

[26] Organisation for Economic Co-operation and Development. OECD Guidelines on the Protection of Privacy and Transborder Flows of Personal Data. http://www.oecd.org/document/18/0,2340,en $264934255 \quad 1815186 \quad 1 \quad 1 \quad 1 \quad 1,00 . h t m l$

[27] Federal Trade Commission, Fair Information Practice Principles. https://www.ftc.gov

[28] Goehring B, Rossi F, Zaharchuk D. Advancing AI ethics beyond compliance. IBM Corporation, April 2020.

[29] The IBM Approach to AI. https://www.ibm.com/se-en/artificial-intelligence/ethics

[30] Ethics and governance of artificial intelligence for health: WHO guidance. Geneva: World Health Organization; 2021. Licence: CC BY-NC-SA 3.0 IGO

[31] Institute of Electrical and Electronics Engineers (IEEE). Global Initiative on Ethics of Autonomous and Intelligent Systems. Ethically Aligned Design: A Vision for Prioritizing Human Well-being with Autonomous and Intelligent Systems, First Edition. IEEE; 2019.

[32] The IEEE Global Initiative on Ethics of Autonomous and Intelligent Systems-faq-11.22.2020

[33] IEEE Ethics in Action in Autonomous and Intelligent Systems_The IEEE 7000 Series

[34] Japan Science and Technology Agency, National Institute of Advanced Industrial Science and Technology, Council on Competitiveness-Nippon, July 25, 2017.

[35] Bossmann J. Top 9 ethical issues in artificial intelligence. World Economic Forum, Oct 21, 2016. https://www.weforum.org/.../top-10-ethical-issues-in-artificial-intelligence/

[36] Proposal for a Regulation of the European Parliament and of the Council laying down harmonized rules on Artificial Intelligence (Artificial Intelligence Act) and amending certain Union legislative acts. https://op.europa.eu/en/publication-detail/-/publication/e0649735-a372-11eb-958501aa75ed71a1/language-en/format-PDF/source-205836026

[37] Google. Artificial Intelligence at Google: Our Principles. https://ai.google/principles/

[38] Future of Life Institute. Asilomar AI Principles. https://futureoflife.org/ai-principles/

[39] https://lawrence.house.gov/media-center/press-releases/brenda-lawrence-and-ro-khanna-introduceresolution-calling-ethical

[40] British Standards Institute. BS 8611:2016 Robots and robotic devices. Guide to the ethical design and application of robots and robotic systems. London: BSI; April 2016.

[41] Blobel B, Ruotsalainen P, Brochhausen M, Oemig F, Uribe GA. Autonomous Systems and Artificial Intelligence in Healthcare Transformation to 5P Medicine - Ethical Challenges. Stud Health Technol Inform. 2020; 270: 1089-1093.

[42] Botelho B. cognitive computing. TechTarget, EnterpriseAI. https://searchenterpriseai.techtarget.com/definition/cognitive-computing

[43] Glasscock J. Cognitive Take on RNA Elevates Biomarker Development, Advances Precision Medicine. Home Magazine December 2019 Vol. 39 No. 12

[44] International Organisation for Standardisation. ISO 23903:2021 Health informatics - Interoperability and integration reference architecture - Model and framework. ISO: Geneva; 2021.

[45] IEEE P7007 - IEEE Draft Ontological Standard for Ethically Driven Robotics and Automation Systems. https://standards.ieee.org/project/7007.html

[46] Almeida MB, Slaughter L, Brochhausen M. Towards an ontology of document acts: Introducing a document act template for healthcare. OTM 2012 Workshops, LNCS 7567, Berlin, New York, Heidelberg, 2012: 420-5.

[47] Brochhausen M, Almeida MA, Slaughter L. Towards a formal representation of document acts and the resulting legal entities. In: Ingthorsson, R.D., Svennerlind, C., and Almäng J (eds.) Johanssonian Investigations. Ontos, Frankfurt, 2013, 120-139.

[48] Watters A. 5 Ethical Issues in Technology to Watch for in 2021. CompTIA, 1 July 2021. https://connect.comptia.org/blog/ethical-issues-in-technology

[49] ACP Says Profit Motive in Medicine May Contribute to a Broken Health Care System. American College of Physicians, https://www.acponline.org

[50] Gilman SC. Ethics Codes and Codes of Conducts as Tools for Promoting an Ethical and Professional Public Services - Comparative Successes and Lessons. World Bank, Washington 2005.

[51] Mittelstadt B. Principles alone cannot guarantee ethical AI. Nature Machine Intelligence November 2019; 1: 501-507. 